Canad. Math. Bull. Vol. 20 (3), 1977

\title{
A CHARACTERIZATION OF THE LEBESGUE INTEGRAL
}

BY

B. S. THOMSON

The first general integrability criterion is due to Riemann. He observed that a necessary and sufficient condition for a function $f(x)$ on $[a, b]$ to be integrable in his sense is that for each $\varepsilon>0$ there exists a $\delta>0$ so that

$$
\sum_{i=1}^{n} \omega\left(f,\left[x_{i-1}, x_{i}\right]\right)\left(x_{i}-x_{i-1}\right)<\varepsilon
$$

where $\left\{x_{0}, x_{1}, \ldots, x_{n}\right\}$ is a partition of $[a, b]$ with diameter less than $\delta$ (and $\omega(f, I)$ denotes the oscillation of $f$ on the interval $I)$.

This conveniently relates integrability to continuity (if only on sets that are large in some sense). The same observation holds for Lebesgue's integral, that integrability is equivalent to a type of continuity, but no direct parallel with the Riemann criterion has been available. In this paper we show that the concept of absolute integrability, introduced by E. J. McShane [3] in 1969, provides this link. Moreover this shows that McShane's concept characterizes Lebesgue integration, a fact which has been well-known but not apparently ever directly established.

0. Preliminaries. In this section we collect some of the basic definitions, notations and results needed in the paper.

0.1 . A partition of an interval $[a, b]$ is a collection

$$
P=\left\{\left(I_{k}, x_{k}\right): k=1,2, \ldots, n\right\}
$$

where $\left\{I_{k}\right\}$ is a collection of non-overlapping closed intervals whose union is $[a, b]$ and each $x_{k} \in I_{k}$.

0.2 . By a $\delta$-fine partition, where $\delta$ is a positive function on $[a, b]$, is meant a partition $P$ satisfying $I \subset(x-\delta(x), x+\delta(x))$ for each $(I, x) \in P$. If $\delta(x)=\delta_{0}>0$ is a constant function on $[a, b]$ we can refer to this as a $\delta_{0}$-fine partition without introducing confusion.

0.3. $m(I)$ denotes the length of the closed interval $I$.

0.4. $\chi(x, E)$ denotes the characteristic function of the set $E$.

0.5 . For a given positive function $\delta$, an arbitrary function $g$ on $[a, b]$ and a set $E \subset[a, b]$, we define

$$
g m_{\delta}(E)=\sup _{P} \sum\{|g(x)| \chi(x, E) m(I):(I, x) \in P\}
$$

Received by the editors December 5, 1975 and, in revised form, July 24, 1976. 
where the supremum is with regard to all $\delta$-fine partitions $P$ of $[a, b]$. (Note that for any $\delta$ a compactness argument shows that such partitions always exist. Also we will abbreviate the summation notation $\sum\{:(I, x) \in P\}$ to $\sum_{P}$.)

0.6 . As in 0.5 we define

$$
g m^{*}(E)=\inf _{\delta} g m_{\delta}(E)
$$

where the infimum is with regard to all positive functions $\delta$ on $[a, b]$.

0.7. It can be shown ( $c f .[6])$ that $g m^{*}$ is always a regular outer measure and that $m^{*}$ is Lebesgue outer measure on $[a, b]$. If $g$ is summable then in fact $g m^{*}(E)=\int_{E}|g(x)| d x$ for measurable $E$.

0.8. A function $g$ on $[a, b]$ is said to be integrable if there is a number $\alpha$ so that for every $\varepsilon>0$ there exists a positive function $\delta$ such that $\mid \sum_{p} g(x) m(I)-$ $\alpha \mid<\varepsilon$ for every $\delta$-fine partition $P$ of $[a, b]$. We can write $\int_{a}^{b} g(x) d m=\alpha$.

1. The Riemann integral. The integral outlined in the preliminary section is equivalent to the Denjoy-Perron integral; this formulation and the relation to the classical integrals is due to R. Henstock [1]. If we follow the details in the case where $\delta(x)$ is restricted to be a constant function then the resulting integral is very obviously the usual Riemann integral. The details on the measure $m^{*}$ similarly shift to show that it is equivalent to the Peano-Jordan content.

We show that McShane's concept of absolute integrability in this context characterizes the Riemann integral and directly corresponds to the Riemann criterion.

Definition (McShaive [3]). A function $f$ defined everywhere on $[a, b]$ is said to be absolutely integrable (Riemann sense) if for every $\varepsilon>0$ there is a positive number $\delta_{0}$ such that

$$
\sum_{(I, x) \in P(J, y) \in P^{\prime}}|f(x)-f(y)| m(I \cap J)<\varepsilon
$$

for every pair $P, P^{\prime}$ of $\delta_{0}$-fine partitions of $[a, b]$.

It is easy to show that such a function is necessarily Riemann integrable (since a Cauchy criterion for the Riemann sums is obtained); it is less obvious that this apparently restrictive condition characterizes the Riemann integral.

Theorem. A function $f$ defined on $[a, b]$ is absolutely integrable (Riemann sense) if and only if it satisfies the Riemann criterion.

The proof is elementary and is omitted. This establishes that for the Riemann case integrability is equivalent to absolute integrability. 
2. The Lebesgue integral. The notion of absolute integrability in the Riemann setting does not serve to restrict the integral. In the more general setting it is much more interesting: while the integral itself is the DenjoyPerron integral, the absolute integrability concept serves to characterize the less encompassing Lebesgue integral. We prove this by a direct assault on the definition which clearly shows its measure-theoretic associations.

Definition. A function $f$ defined everywhere on $[a, b]$ is said to be absolutely integrable if for every $\varepsilon>0$ there is a positive function $\delta$ such that

$$
\sum_{(I, x) \in \mathbf{P}(J, y) \in \mathbf{P}^{\prime}}|f(x)-f(y)| m(I \cap J)<\varepsilon
$$

for every pair $\left(P, P^{\prime}\right)$ of $\delta$-fine partitions of $[a, b]$.

THEOREM. A function defined everywhere on $[a, b]$ is absolutely integrable if and only if $f$ is measurable and $f m^{*}$ is finite.

Proof. Let us prove the sufficiency part firstly on the assumption that $|f(x)| \leq M$ everywhere. Then using Lusin's criterion for measurability we may choose an open set $G$ with $m^{*}(G)<\varepsilon / 8 M(b-a)$ and so that $f$ is continuous restricted to $F=[a, b] \backslash G$. Choose $\delta_{1}(x)$ so that if $x \in G$ then $\left(x-\delta_{1}(x), x+\delta_{1}(x)\right) \subset G$ and if $x \in F$ then $|f(x)-f(y)|<\varepsilon / 2(b-a)$ if $|x-y|<$ $\delta_{1}(x)$ with $y \in F$. Let $\delta(x) \leq \delta_{1}(x)$ so that $m_{\delta}(G)<2 m^{*}(G)<\varepsilon / 4 M(b-a)$.

Then if $P$ and $P^{\prime}$ are arbitrary $\delta$-fine partitions of $[a, b]$ it is a routine matter to compute that

$$
\sum_{(I, x) \in \mathbb{P}(J, y) \in \mathbb{P}^{\prime}}|f(x)-f(y)| m(I \cap J) \leq \frac{\varepsilon}{2(b-a)} \sum \sum m(I \cap J)+2 M m_{\delta}(G)<\varepsilon
$$

by considering separately sums with both $x$ and $y$ in $F$ and sums with one at least of $x$ or $y$ in $G$.

Suppose now that $f$ is measurable and $(f m)^{*}$ is finite: if we construct $f_{N}(x)=f(x)$ if $|f(x)| \leq N$ and zero otherwise then it is possible to show that $\left(f-f_{N}\right) m^{*} \rightarrow 0$. (Indeed this is equivalent to $f m^{*}\left(X_{n}\right) \rightarrow 0$ where $X_{n}=$ $\{x:|f(x)|>n\}$ and so follows from standard arguments in measure theory.) Hence given $\varepsilon>0$ choose an $N$ so that $\left(f-f_{N}\right) m^{*}[a, b]<\varepsilon / 4$ and since $f_{N}$ is bounded and measurable we may, by the preceeding, choose $\delta_{1}$ so that

$$
\sum_{P} \sum_{P^{\prime}}\left|f_{N}(x)-f_{N}(y)\right| m(I \cap J)<\varepsilon / 2
$$

for any $\delta_{1}$-fine partitions $P$ and $P^{\prime}$. We choose a $\delta \leq \delta_{1}$ so that

$$
\left(f-f_{N}\right) m_{\delta}[a, b]<2\left(f-f_{N}\right) m^{*}[a, b]<\varepsilon / 2
$$


and then we have for any $\delta$-fine partitions $P$ and $P^{\prime}$

$$
\begin{aligned}
\sum_{P} \sum_{P^{\prime}}|f(x)-f(y)| m(I \cap J) \leq & \sum \sum\left|f(x)-f_{N}(x)\right| m(I \cap J) \\
& +\sum \sum\left|f_{N}(x)-f_{N}(y)\right| m(I \cap J) \\
& +\sum \sum\left|f_{N}(y)-f(y)\right| m(I \cap J) \\
\leq & 2\left(f-f_{N}\right) m_{\delta}[a, b]+\varepsilon / 2<\varepsilon
\end{aligned}
$$

as required and so $f$ is absolutely integrable.

To establish the necessity of the conditions it is easy firstly to show that $\mathrm{fm}^{*}$ is finite by fixing $P$ in the definition and letting $P^{\prime}$ vary. To obtain that $f$ is measurable we use another famous characterization of measurability, namely that it is equivalent to a.e. approximate continuity (see for example Saks [5]).

Let $F_{n}(y)=\{x:|f(x)-f(y)| \geq 1 / n\}$ and $E_{n}=\{x: x$ is a point of density for $\left.F_{n}(x)\right\}$. It is enough to prove that each $E_{n}$ is a set of measure zero to complete the proof of the theorem. Given $\varepsilon>0$ choose $\delta_{1}$ so that for any $\delta_{1}$-fine partitions $P$ and $P^{\prime}$;

$$
\sum_{P} \sum_{P^{\prime}}|f(x)-f(y)| m(I \cap J)<\varepsilon / 8 n
$$

and $\delta_{2}$ so that

$$
\frac{m^{*}\left(F_{n}(x) \cap I\right)}{m^{*}(I)}>\frac{1}{2}
$$

for all $x \in E_{n}$ and intervals $I$ with $x \in I \subset\left(x-\delta_{2}(x), x+\delta_{2}(x)\right)$.

If $\delta=\min \left(\delta_{1}, \delta_{2}\right)$ then there exists a $\delta$-fine partition $P=\left\{\left(I_{k}, x_{k}\right)\right\}$ with

$$
\sum_{k} \chi\left(x_{k}, E_{n}\right) m\left(I_{k}\right) \geq \frac{1}{2} m_{\delta}\left(E_{n}\right) \geq \frac{1}{2} m^{*}\left(E_{n}\right) .
$$

But as $\delta \leq \delta_{2}$ we have

$$
m^{*}\left(E_{n}\right) \leq 4 \sum \chi\left(x_{k}, E_{n}\right) m^{*}\left(F_{n}\left(x_{k}\right) \cap I_{k}\right) .
$$

We can choose a $\delta$-fine partition $P^{\prime}=\left\{\left(J_{i}, y_{i}\right)\right\}$ so that for each $k$

$$
m^{*}\left(F_{n}\left(x_{k}\right) \cap I_{k}\right) \leq 2 \sum_{i} \chi\left(y_{i}, F_{n}\left(x_{k}\right)\right) m\left(I_{k} \cap J_{i}\right)
$$

Combining we have

$$
m^{*}\left(E_{n}\right) \leq 8 \sum_{i} \sum_{k} \chi\left(x_{k}, E_{n}\right) \chi\left(y_{i}, F_{n}\left(x_{k}\right)\right) m\left(I_{k} \cap J_{i}\right)
$$


but whenever $x_{k} \in E_{n}$ and $y_{i} \in F_{n}\left(x_{k}\right)$ we have $\left|f\left(x_{k}\right)-f\left(y_{i}\right)\right| \geq 1 / n$ so that

$$
m^{*}\left(E_{n}\right) \leq 8 n \sum_{i} \sum_{k}\left|f\left(x_{k}\right)-f\left(y_{i}\right)\right| m\left(I_{k} \cap J_{i}\right)<\varepsilon
$$

by choice of $\delta \leq \delta_{1}$. Since $\varepsilon>0$ was arbitrary $m^{*}\left(E_{n}\right)=0$ completing the proof.

\section{REFERENCES}

1. R. Henstock, The equivalence of generalized forms of the Ward, variational, Denjoy-Stieltjes and Perron-Stieltjes integrals, Proc. London Math. Soc. (3), 10 (1960), pp. 281-303.

2. R. Henstock, A Riemann-type integral of Lebesgue power, Canad. J. Math. 20 (1968), pp. 79-87.

3. E. J. McShane, A Riemann-type integral that includes Lebesgue-Stieltjes, Bochner and Stochastic integrals, Memoirs of the Amer. Math. Soc. No. 88, 1969.

4. E. J. McShane, A unified theory of integration, Amer. Math Monthly, (80) no. 4 (1973), pp. 349-359.

5. S. Saks, Theory of the Integral, 2nd English edn. (Warsaw, 1937).

6. B. S. Thomson, A theory of integration, Duke Math J., vol. 39 (1972), pp. 503-510.

DEPT. OF MATHS.,

Simon Fraser UNIVERSITY,

BURNABY, B.C.

CANADA V5A 1 S6 